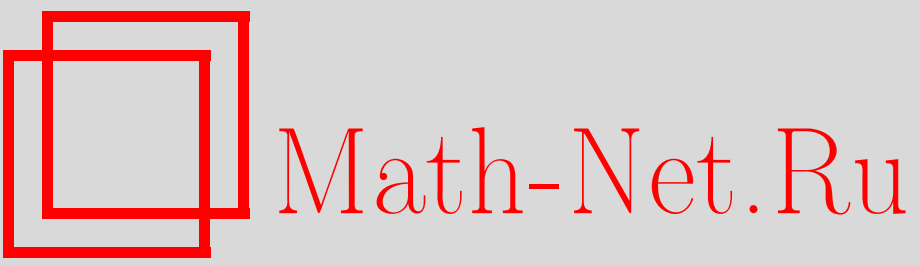

М.-М. Деза, М. И. Штогрин, Вложение графов в гиперкубы и кубические решетки, УМН, 1997, том 52, выпуск 6, 155-156

DOI: https://doi.org/10.4213/rm899

Использование Общероссийского математического портала Math-Net.Ru подразумевает, что вы прочитали и согласны с пользовательским соглашением

http://www.mathnet.ru/rus/agreement

Параметры загрузки:

IP : 35.173 .137 .237

26 апреля 2023 г., 16:47:18

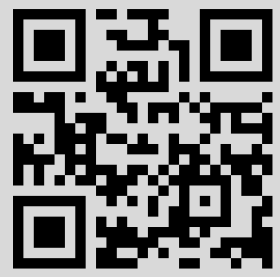




\title{
ВЛОЖЕНИЕ ГРАФОВ В ГИПЕРКУБЫ И КУБИЧЕСКИЕ РЕШЕТКИ
}

\author{
М. ДЕзА, М. И. Штогрин
}

Мы продолжаем исследования, начатые в [1]; оттуда взяты определения и обозначения.

1. Правильных разбиений $S^{n}, R^{n}, H^{n}$ на компактные правильные многогранники всего имеется по 11 при $n=3$ и $n=4$ и 4 при любом $n \geqslant 5$. Ниже мы увидим, что при $n=3$ и $n=4$ не вложимы лишь по 6 разбиений: 24, 600, 120, $($ Ico, Do $),\left(D o, \beta_{3}\right),(D o, I c o)$ и $\left(\alpha_{4}, 600\right),\left(\beta_{4}, 24\right)$, $\left(24, \gamma_{4}\right),\left(120, \alpha_{4}\right),\left(120, \beta_{4}\right),(120,600)$. Символы $\alpha_{n}, \beta_{n}, \gamma_{n}, \delta_{n}, I c o, D o, 24,120,600$ обозначают $n$-симплекс, $n$-октаэдр, $n$-куб, $Z_{n-1}$, икосаэдр, додекаэдр, 4 -мерный 24 -, 120 -, 600 -гранник; $(F, V)$ - правильный многогранник с гранью $F$ и звездой $V$.

Теорема 1. При $n=3$ и $n=4$ вложимыми правильными разбиениями, кроме $\alpha_{n+1}$, $\beta_{n+1}, \gamma_{n+1}, \delta_{n+1}$, являются лишь разбиения $H^{n}$ на кубы $\gamma_{n}$, m.е. $\left(\gamma_{3}\right.$, Iсо $) \rightarrow Z_{\infty}$, $\left(\gamma_{4}, 600\right) \rightarrow Z_{\infty}$.

Вложения и ссылки для случаев $n<4$ и $n>5$ указаны в [1]. При $n \geqslant 5$ дуальньй полу- $n$-кубу многогранник не 5-гонален. Вложение $(2 p, 2 q) \rightarrow Z_{\infty}$ имеет широкое обобщение: любое разбиение $H^{2}$ на четноугольники с четным схождением (и дуальное) вложимо в $Z_{\infty}$. Кроме не 7-гональных 600 и $\left(\alpha_{4}, 600\right)$, остальные невложимые правильные разбиения не 5 -гональны. Не 7 -гональность 600 , доказанная впервые в [2], следует даже прямо из конструкции Госсета: добавить пирамиды на все 24 икосаэдральные грани полурегулярного многогранника $s\{3,4,3\}$; пирамида на икосаэдре 5-, но не 7-гональна. Вложение $s\{3,4,3\} \rightarrow \frac{1}{2} H_{12}$ подтвердилось. Многогранник 600 , кажется, 5-гонален.

Теорема 2. Усеченные разбиения $\operatorname{tr} P$ 5-гональны (более того, вложимы) только для следующих правильных разбиений: $\operatorname{tr} 24 \rightarrow H_{12}, \operatorname{tr}\left(24, \gamma_{4}\right) \rightarrow Z_{12}, \operatorname{tr}\left(p^{q}\right)$ при $\left(p^{q}\right) \neq$ $\left(3^{5}\right)$ u $q \neq 3\left(\right.$ включая $\operatorname{tr} \beta_{3} \rightarrow H_{6}, \operatorname{tr}\left(3^{6}\right)=\left(6^{3}\right) \rightarrow Z_{3}, \operatorname{tr} \delta_{3}=\left(4.8^{2}\right) \rightarrow Z_{4}$, гиперболические $\operatorname{tr}\left(p^{q}\right) \rightarrow Z_{\infty}$ при $p=2 k$ или $\operatorname{tr}\left(p^{q}\right) \rightarrow \frac{1}{2} Z_{\infty}$ nри $p=2(k+1)$ c $\left.q>3\right)$.

Здесь $\left(24, \gamma_{4}\right)=V o\left(D_{4}\right)$, где $V o(\Lambda)$ означает остов разбиения Вороного для решетки $\Lambda$.

Разбиения $(\operatorname{tr} P)^{*}$ получаются из $P^{*}$ добавлением пирамид над всеми $(n-1)$-гранями. $(\operatorname{tr} P)^{*} \rightarrow \frac{1}{2} H_{m}, m=7,12,10,26$, если $P=\alpha_{3}, \gamma_{3}, I c o, D o .\left(\operatorname{tr}\left(p^{q}\right)\right)^{*} \rightarrow \frac{1}{2} Z_{3}$ или $\frac{1}{2} Z_{\infty}$ и не 5 -гонально, если $\left(p^{q}\right)=\left(3^{6}\right)$ или $\left(6^{3}\right)$ и $\left(4^{4}\right),\left(3^{4}\right)$. $(\operatorname{tr} P)^{*}$ не 5-гонально для $P=$ $24,600,120, \alpha_{n}, \beta_{n}, \gamma_{n}, \delta_{n}, n \geqslant 4$. Гиперболические $\left(\operatorname{tr}\left(p^{q}\right)\right)^{*}$ не 5-гональны при $q=4$, вложимы в $\frac{1}{2} Z_{\infty}$ при $q \neq 4$.

Для усечений до середины ребер имеем: $\operatorname{Tr} \alpha_{n} \rightarrow \frac{1}{2} H_{n+1}, \operatorname{Tr} \beta_{n}$ и $\operatorname{Tr} \gamma_{n}$ не 5-гональны при $n \geqslant 3, \operatorname{Tr}\left(p^{q}\right) \rightarrow Z_{\infty}$ при $p>3<q$ (оба четные) и $\operatorname{Tr}\left(p^{q}\right) \rightarrow \frac{1}{2} Z_{\infty}$ при остальных $p>3<q$, не 5-гонально при $p=3<q$ четно или четно $p>3=q$.

Отметим аналогию вложения обычных усечений $\alpha_{2}, \beta_{3}, 24, V o\left(D_{4}\right)$ в $H_{3}, H_{6}, H_{12}, Z_{12}$ и их золотых усечений $C_{3}, I c o, s\{3,4,3\}, s\{3,4,3,3\}$ в $\frac{1}{2} H_{3}, \frac{1}{2} H_{6}, \frac{1}{2} H_{12}$ и, быть может, $\frac{1}{2} Z_{12}$.

2. Как известно [3], невыпуклые (звездные) правильные многогранники не существуют для $S^{n}$ при $n \geqslant 5$ и для $R^{n}$ при всех $n$; при $n=4$ их 10 , при $n=3$ их 4 . Имея остов обычного додекаэдра, большой звездньй додекаэдр $\left\{\frac{5}{2}, 3\right\}$ вложим в $\frac{1}{2} H_{10}$. Имея остов икосаэдра, остальные $\left\{3, \frac{5}{2}\right\}$, $\left\{\frac{5}{2}, 5\right\},\left\{5, \frac{5}{2}\right\}$ вложимы в $\frac{1}{2} H_{6}$. Здесь $\frac{5}{2}$ обозначает звездный 5 -угольник.

Существует 6 бесконечных правильых многогранников: 3 выпуклых $\delta_{3}=Z_{2},\left(3^{6}\right),\left(6^{3}\right)$ и 3 невыпуклых. Последние (см., например, [4, с. 105]) являются замкнутыми лабиринтами: 1$) \delta_{4}$ с половиной выброшенных кубов; 2$) V o\left(A_{3}^{*}\right)$ с половиной выброшенных усеченных $\left.\beta_{3} ; 3\right)$ униформное разбиение Фоппля на $\alpha_{3}$ и усеченные $\alpha_{3}$ с половиной выброшенных $\alpha_{3}$ (одной ориентации).

Теорема 3. Остовы лабиринтов 1) и 2) вложимы, соответственно, в $Z_{3}$ и $Z_{6}$. Остов лабиринта 3) не 5-гонален.

Примеры 4 интересных невьпуклых многогранников даны, например, в [4, с. 98, 248, 161, 34]: а) 7-гранник $P$, полученньй из $\beta_{3}$ заменой 4 из 8 его граней, взятых через одну, на 3 квадрата,

Работа выполнена при финансовой поддержке Российского фонда фундаментальных исследований (грант № 96-01-00166). 
представляет собою проективную плоскость; остовы $P$ и $\beta_{3}$ совпадают и вложимы в $\left.\frac{1}{2} H_{4} ; \mathrm{b}\right)$ имея остов $\left(\operatorname{tr} \gamma_{3}\right)^{*}$, т.е. $\beta_{3}$ с пирамидами на каждой грани, стелла октангула вложима в $\frac{1}{2} H_{12} ;$ c) имея остов икосаэдра, ортогоналшный икосаэдр Джессена (он нетвердый) вложим в $\frac{1}{2} H_{6} ; \mathrm{d}$ ) полярньй коллапсоид не 5-гонален.

3. Назовем шамферингом планарного графа $G$ и обозначим через $\mathrm{Ch} G$ замену каждого его ребра шестиугольником: над всеми элементарными ячейками, вырезаемыми графом на плоскости или сфере, строятся призмы, а затем все исходные ребра исключаются.

Теорема 4. Все вложимые шамферинги правильных планарных разбиений суть для $S^{2}: \mathrm{Ch} \gamma_{3} \rightarrow H_{7} u \mathrm{Ch} D o \rightarrow \frac{1}{2} H_{22} ;$ для $R^{2}: \operatorname{Ch}\left(4^{3}\right) \rightarrow Z_{4} u \mathrm{Ch}\left(6^{3}\right)=\left(6^{3}\right) \rightarrow Z_{3} ;$ для $H^{2}: \operatorname{Ch}(2 m+1, q) \rightarrow \frac{1}{2} Z_{\infty}$ npu $m \neq 1 u \mathrm{Ch}(2 m, q) \rightarrow Z_{\infty}$

Следует отметить, что 1) шамферинги всех остальных правильных разбиений, а также полуправильных разбиений $S^{2}, R^{2}$ не 5-гоналњны; 2) кроме $\mathrm{Ch}_{t}\left(6^{3}\right)=\left(6^{3}\right)$, итеративные шамфоринги всех других правильных разбиений не 5-гональны; 3$)$ кроме $\left(\mathrm{Ch} \alpha_{3}\right)^{*} \rightarrow \frac{1}{2} H_{8},(\mathrm{Ch} P)^{*}$ для других правильных разбиений $S^{2}$ и $R^{2}$ не 5-гональны; 4) не 5-гональность $\mathrm{Ch} P$ вытекает из того, что $P$ имеет либо треугольную грань, либо, как изометрический подграф̆ остова, 4 -угольную грань с висячими ребрами на двух противоположных вершинах; 5) $\mathrm{Ch} \gamma_{3}, \mathrm{Ch}\left(4^{4}\right), \mathrm{Ch}(2 m, q)$ зонотопальны. $\mathrm{Ch}\left(4^{4}\right)$ дуален разбиению $\left(4^{4}\right)$, рассматриваемому как бесконечная шахматная доска, с пирамидами на всех черных клетках. $\mathrm{Ch}\left(4^{4}\right)$ был на мозаике $\mathrm{XV}$ века в Португалии (см. $[5$, рис. 9,10$])$.

Все 22 эквитранзитивные разбиения $R^{2}$ (их конгруэнтные грани принадлежат одной и той же орбите группы автоморфизмов разбиения) даны в [6]; это все 3 правильных, 7 архимедовых (все зеркально-симметричные) и 12 других, включая семь 2-униформных, четыре 3-униформных и одно 4-униформное. О вложимости первых 10 см. в [1].

Теорема 5. Среди последних 12 әквитранзитивных разбиений $R^{2}$ и им дуальных 5-гональны (более того, вложимы) только следующие: $\left(3^{4} \cdot 6 ; 3^{2} \cdot 6^{2}\right) \rightarrow \frac{1}{2} Z_{5}$, $\left(3^{3} .4^{2} ; 4^{4}\right)_{1} \rightarrow \frac{1}{2} Z_{3}, \quad\left[3^{6} ; 3^{2} .6^{2}\right] \rightarrow Z_{\infty}, \quad\left[3.4^{2} .6 ; 3.6 .3 .6\right]_{2} \rightarrow Z_{3}, \quad\left(3^{6} ; 3^{2} .6^{2} ; 6^{3}\right) \rightarrow \frac{1}{2} Z_{6}$, $\left(3^{4} .6 ; 3^{2} .6^{2} ; 6^{3}\right) \rightarrow \frac{1}{2} Z_{5},\left[3.4^{2} .6 ; 3.4 .6 .4 ; 4^{4}\right] \rightarrow Z_{4},\left[3^{6} ; 3^{2} .6^{2} ; 6^{3} ; 6^{3}\right]_{1} \rightarrow \frac{1}{2} Z_{\infty}$.

Заметим, что следующие два разбиения $R^{2}$ (указанные, например, в $[4$, с. 104,89$]$ ) не 5-гональны: а) разбиение Дюрера на правильные 5 -угольники и ромбы; b) разбиение на греческие кресты. (Это случай $t=2$ разбиения $\left(4^{4}\right)$ с $t$ новыми точками на каждом ребре; только при $t=0$ получается 5-гональность.) Разбиение Пенроуза $R^{2}$ (и его аналог в $R^{3}$ ) двумя золотьми ромбами (ромбоэдрами) вложимо в $Z_{5}\left(Z_{6}\right)$, но его подразделение Робинсона и другое разбиение на равнобедренные золотые треугольники (см. [7, рис. 2]), а также pinwheel-разбиение на $1-2-\sqrt{5}$ прямоугольные треугольники (см. [8]), не 5-гональны.

\section{СПИСОК ЛИТЕРАТУРЫ}

[1] Деза М., Штогрин М. И. // УМН. 1996. Т. 51. №6. С. 199-200. [2] Assouad P. // The Geometric Vein, the Coxeter Festschrift. Berlin: Springer, 1981. P. 141-147. [3] Coxeter H.S. M. Regular Polytopes. New York: The Macmillan Company; London: Collier-Macmillan Ltd., 1963. [4] Wells D. The Penguin Dictionary of curious and interesting geometry. New York: Penguin Books, 1991. [5] Grünbaum B., Shephard G. C. Tilings and Patterns. New York: W. H. Freeman and Company, 1987. [6] DeBroey I., Landuyt F. // Geom. Dedicata. 1981. V. 11. P. 47-60. [7] Baake M., Schlottmann M. // Proc. 5th Conf. on Quasicrystals, 1995. P. 15-21. [8] Radin C. // Ann. Math. 1994. V. 139. P. 661-702.

LIENS, Ecole Normale Supérieure and CNRS, Paris; Математический институт им. В. А. Стеклова РАН
Принято редколлегией 03.09.1997 